\title{
Selected effects of VTI anisotropy on downhole microseismic data
}

\author{
Eryk Święch, Andrzej Pasternacki, Tomasz Maćkowski
}

AGH University of Science and Technology, Faculty of Geology, Geophysics and Environmental Protection, Department of Fossil Fuels; al. A. Mickiewicza 30, 30-059 Krakow, Poland; e-mail:swiech.eryk@gmail.com,anpaster@agh.edu.pl,mackowsk@agh.edu.pl

(C) 2016 Authors. This is an open access publication, which can be used, distributed and reproduced in any medium according to the Creative Commons CC-BY 4.0 License requiring that the original work has been properly cited.

Shale gas is one of the well-known unconventional resources of natural gas all over the world. This term refers to natural gas that is trapped within shale formations. Shales are fine - grained sedimentary rocks which can be reach resources of both petroleum and natural gas. This sedimentary rocks are heavily layered and in their nature exhibit VTI velocity anisotropy behavior (Van Dok et al. 2011). This statement indicates that the world among us is not isotropic and we should not neglect this fact in our geophysical research.

Anisotropy, in general is the property of the material. It can be described as the attribute of a material's property with respect to the direction in which it is measured (Pereira \& Jones 2010). There are two essential types of anisotropy: VTI and HTI. Vertical velocity layering gives rise to VTI (vertical transverse isotropy) velocity in which seismic wave velocity is faster in the horizontal direction than in the vertical one. The second type of isotropy is horizontal transverse isotropy (HTI) which causes azimuthal traveltime variations. The common mechanism for this type of anisotropy is vertical aligned fractures in an isotropic background medium (Jenner 2011.)

Authors of this study focused mostly on VTI as this type of anisotropy is present in shale formations, as a result of small scaled heterogeneities from fine layering (Thomsen 1986). The VTI anisotropy can be mathematically described by using three Thomsen parameters: epsilon, delta and gamma. Epsilon is a measure of the difference between the horizontal and vertical propagation velocities for compressional waves. Gamma parameter is a measure of the difference in the horizontal and vertical propagation velocities for horizontally polarized shear waves (SH waves). Delta parameter is not easily described either mathematically or qualitatively (Pereira \& Jones 2010), but it influences the anisotropy velocities in medium incidence angles. These parameters can be mathematically expressed by equations proposed by Leon Thomsen (Thomsen 1986).

In this study, authors present influence of VTI anisotropy on microseismic data recorded during hydraulic fracturing of shale intervals in one of the well located in Northern Poland. Authors points out how the anisotropy affects on microseismic events location, locating them in isotropic and anisotropic velocity models with usage of TGS algorithm. Furthermore, authors indicate possible solution to estimate VTI parameters based on microseismic data.

VTI anisotropy parameters plays critical role not only in case of microseismic data analysis but also in processing of active seismic data. Authors proved that VTI anisotropy present in the investigated area has strong influence on microseismic events location especially in depth. Moreover estimation of VTI anisotropy parameters based on microseismic data with usage of Thomsen equations is possible. 
This study was supported by the Grant of The Polish National Center for Research and Development in the Program Blue Gas, project entitled "Appraisal of microseismic monitoring techniques of hydraulic fracturing and development of optimal processing and interpretation methodologies" (Acronym: SHALEGASMICROS). Authors would like to thank GeoTomo for research and education grant of $\mathrm{MiVu}^{T M}$ Microseismic Processing System in which calculations were done.

\section{REFERENCES}

Jenner E., 2011. Combining VTI and HTI anisotropy in prestcack time migtation: Workflow and data examples. The Leading Edge, 30, 7, 732-739.

Pereira A.M. \& Jones M., 2010. Fundamental of borehole seismic technology. Schlumberger.

Thomsen L., 1986. Weak elastic anisotropy. Geophysics, 51, 10, 1954-1966.

Van Dok R., Fuller B., Engelbrecht L. \& Sterling M., 2011. Seismic anisotropy in microseismic event location analysis. The Leading Edge, 30, 7, 766-770. 